\title{
Prosthetic Rehabilitation of Crowded Maxillary Anterior Teeth
}

\author{
Emese Rita Markovics', Kinga Dörner'1, Orsolya Birta1', Julia Popa² \\ 1 Department of Removable Prosthetic Dentistry, Faculty of Dental Medicine, University of Medicine and Pharmacy, Tîrgu Mureș, Romania \\ 2 Student, Faculty of Dental Medicine, University of Medicine and Pharmacy, Tîrgu Mureș, Romania
}

\section{CORRESPONDENCE}

Emese Rita Markovics

Str. Gheorghe Marinescu nr. 38

540139 Tîrgu Mureș, Romania

Tel: +40 265215551

E-mail: emesemarkovics@gmail.com

\section{ARTICLE HISTORY}

Received: 2 March, 2017

Accepted: 7 March, 2017
Kinga Dörner • Str. Gheorghe Marinescu nr. 50 540139 Tîrgu Mures, Romania, Tel: +40 265215551. E-mail:

Orsolya Birta • Str. Gheorghe Marinescu nr. 38 540139 Tîrgu Mureș, Romania, Tel: +40 265215551 Julia Popa • Str. Gheorghe Marinescu nr. 38, 540139 Tîrgu Mures, Romania, Tel: +40 265215551

\begin{abstract}
Introduction: In this case report we present the aesthetical correction of crowded anterior teeth in a patient who has refused the orthodontic treatment. We also discuss how this correction can be resolved from the point of view of dental prosthetic and what measurements can be helpful in order to collect the data used to restore a satisfying smile on the patient's face using dental ceramic restoration. Case presentation: A 32-year-old patient complained about his crowded incisors. We examined the incisal curvature, the proportion between the height and the width of the teeth, and the relation between the widths of the teeth and the golden proportion. The results of the measurements were compared to the characteristic values of the ideal denture. After the examination of the dental plaster cast, calculating the difference between the existing space and the necessary space, we were able to calculate the lack of space, which caused the crowded teeth. Conclusion: Along the prosthetic planning, these measurements may serve as a guideline to the dentist as an objective basis for the formation of an aesthetic denture and smile.
\end{abstract}

Keywords: crowded incisors, measurements, ceramic crowns, aesthetics

\section{INTRODUCTION}

It is important that an adequate treatment plan be established before starting a prosthetic treatment. ${ }^{1-4}$ With the help of measurements, we can estimate the condition of the denture before the prosthetic treatment, then comparing the obtained values with the ideal ones, we are able to establish the severity of the case. ${ }^{5}$ Using this type of treatment, we aim to modify these values in such a way that they become closer to the ideal values recorded during the measurements. Thus, we try to achieve the most aesthetic result in a given situation. At the end of the prosthetic treatment, after having done the measurements again, we may draw the conclusions regarding the grade of success of the treatment, moreover, we may establish to what extent we have managed to approach the ideal results. We examine the arch of the smile, the proportion between the height and the width of the teeth, and finally the relation between the widths of the teeth and the golden proportion. ${ }^{5-12}$ Model analysis cannot be omitted in making an exact diagnosis. 


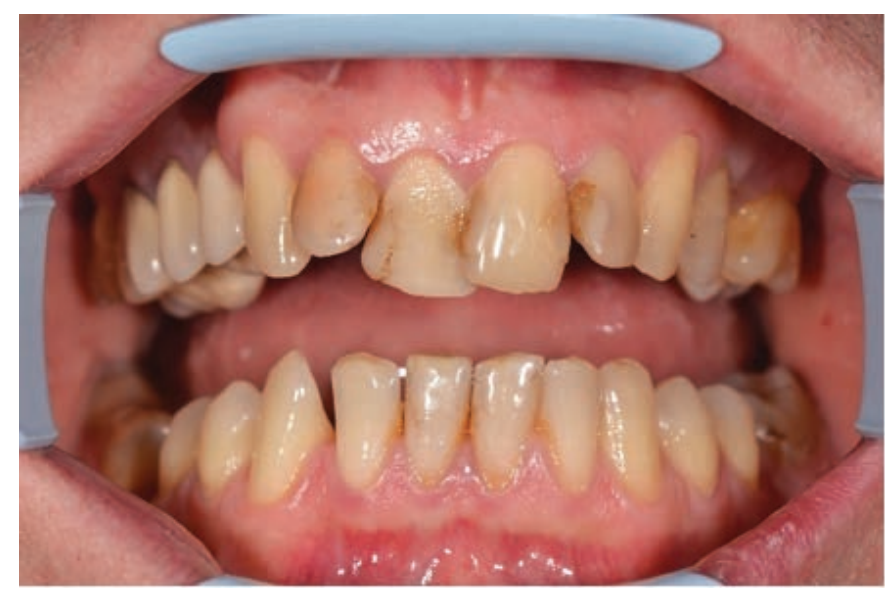

FIGURE 1. Preoperative photograph

\section{CASE REPORT}

A 32-year-old patient was concerned about the aesthetic appearance of his smile, complaining about his crowded and discolored incisors and obturation margins (Figure 1). It was clear that in order to reach an acceptable aesthetic it was not enough to perform only an orthodontic treatment. Taking into account the state of the teeth, a prosthetic rehabilitation was also needed.

On the first appointment, during the examination the following dental anomalies were observed: crowded and slightly rotated upper incisors, deep bite, irregular curvature of the maxillary incisal edge, discolored incisors and obturation margins, the missing upper right first premolar was completed with a metal-ceramic cantilever bridge fixed on the right second premolar and the right first molar. To establish the proper treatment plan, preoperative radiographic and photographic documentation was made, as well as a preliminary impression (alginate) in order to make the study-cast. After a thorough study of this documentation, two treatment options were suggested to the patient. The first option was orthodontic treatment followed by a prosthetic rehabilitation, and the second one was only a prosthetic treatment with full ceramic crowns, which, of course, required a bigger sacrifice of healthy dental tissue in order to make the correction of the crowded teeth. The patient refused the orthodontic treatment, because of the duration and aesthetic drawbacks of the treatment, preferring the second option.

On the next appointments the pre-prosthetic treatment on the superior dental arch followed: the extraction of the left second premolar's root remains, root apex resection of the right lateral incisor, root canal treatments of the central incisors and the left lateral incisor, and the obturation of the right lateral incisor. The patient agreed to the publica- tion of his data and the institution where the patient had been admitted, approved the publication of the case.

Measurements were made on the photos and plaster cast before the prosthetic treatment, and the results were compared to the characteristic values of the ideal denture. During the analysis of the photos, the visible mesiodistal width of the maxillary anterior teeth and their proportion were examined. Because of the curvature of the dental arch, there was a difference between the width of the tooth when viewed frontally and its real width. When analyzing an aesthetic, attractive smile from a frontal view, the best way to determine how a tooth's width compares to the one next to it, is by using the ideal situation provided by the golden proportion. According to this, the visible width of the upper lateral incisor is $62 \%$ of the width of the upper central incisor, while the visible width of the upper canine is, again, $62 \%$ of the width of the upper lateral incisor.

As in our case the four superior incisor teeth were crowded, this proportion was changed: the visible width of the upper right lateral incisor was $86 \%$ of the upper central incisor's width, and the upper left lateral incisor's visible width was $66.6 \%$ of the upper left central incisor's width. In order to get the most aesthetic result, we had to aim to make this value approach the ideal one.

Research has confirmed that the width of the central incisor crown has to be $75-80 \%$ of the height of the crown in an aesthetic denture. This proportion had to be taken into account when planning, because the width of the crown needed to diminished, in order to restore the teeth that were crowded due to the lack of space. We also had to pay attention not to narrow the teeth too much and make them look less aesthetical. It was important to examine the incisal arch when planning; in our case this was uneven, irregular. The ideal incisal arch is parallel with the curve of the lower lip. We have to aim for the formation of a symmetrical incisal arch, which follows the curve of the lower lip. After the examination of the dental plaster cast, calculating the difference between the space at our disposal and the necessary space for the teeth, we could calculate the lack of space we were faced with.

The measurement of the space at our disposal was carried out measuring the wire led from the mesial portion of the upper right first molar to the mesial portion of the upper left first molar, over the vestibular cusps of the premolars, cusps of the canines, as well as over the incisal edge of the incisors. In order to ascertain the necessary space, we measured the mesiodistal width of the premolars, the canines and the incisors. By subtracting the first value from the second one, we calculated how much 


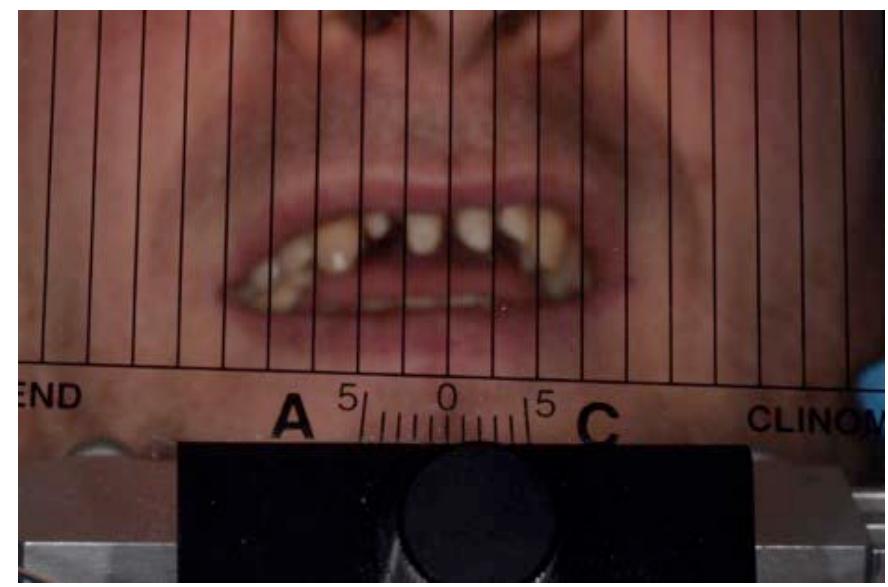

FIGURE 2. Angulation determination

space was missing. From this value we could deduce to what degree we should decrease the width of the crowns, in order for them to fit symmetrically in the teeth arch. In our case, the space at our disposal was $7.4 \mathrm{~cm}$, and the necessary space was $8 \mathrm{~cm}$, the difference between the two being $6 \mathrm{~mm}$. During the prosthetic treatment, the central incisors were prepared for the ceramic crowns. The gingival margin of the preparation was aligned in a juxta-gingival position for easier cleaning and for better aesthetical effect. In order to determine the correct angulation of the anterior teeth alignment, we used a suitable instrument, the Clinometer (Amann Girrbach, Koblach, Austria) attached to the Artex Facebow (Amann Girrbach, Koblach, Austria) (Figure 2.)

The determination of the shade was performed digitally, using the Vita Easyshade system (Vita-Zahnfabrik, Bad Säckingen, Germany): C2 for the crown part, A3 for the cervical part. In order to obtain the desired tooth shade, we also determined the color of the tooth stumps with the help of the IPS Natural Die Material shade guide (Ivoclar Vivadent, Schaan, Liechtenstein).

The gingival displacement before the final impression of the four incisors was made with the double cord technique for a better visualization of the preparation margins. The first thinner cord (00-Elite cord, Zhermack, Badia Polesine, Italy), which was not impregnated, remained in the gingival sulcus during the making of the impression. The second cord was thicker (0-Elite cord, Zhermack), it was impregnated (Gingiva Liquid Roeko, Coltene, Altstätten, Switzerland), and it was taken away before making the impression.

The final impression was made with A-silicone (Elite, Zhermack) with the one step mix technique (final impression), while the impression of the antagonist dental arch was made with C-silicone (Zeta Plus + Oranwash, Zher-

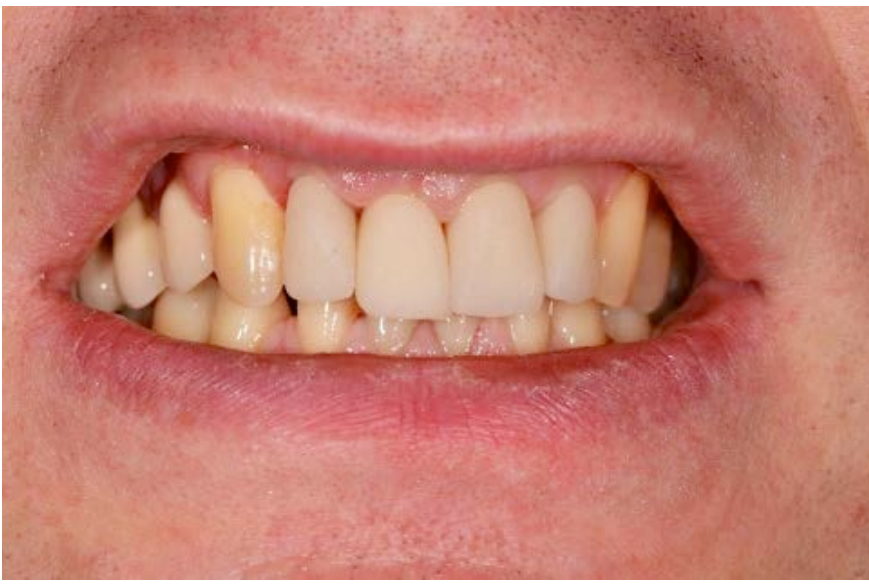

FIGURE 3. Provisional crowns

mack), and the registration of the intermaxillary relations with A-silicone. The provisional crowns (Figure 3) were made from PMMA blocks with CAD-CAM system to ensure a good marginal fit of the crowns. Their bond was made with Temp-Bond NE (Kerr, Orange, CA, USA) temporary dental cement.

The final ceramic crowns were made from IPS e.max press ceramic (Ivoclar Vivadent) (Figure 4). This choice was made due to its excellent characteristics: exceptional translucency corresponding to natural teeth, high mechanical strength, as well as superior fracture toughness. It also presented a superior opalescence/fluorescence and low bacterial adhesion.

On the occasion of the crown try-in the marginal adaptation, the contour and the color were verified. After the preparation of the teeth surface and of the crowns, the bonding of the crowns into place with SR Nexco Paste (Ivoclar Vivadent) composite followed. After checking the occlusion, the final polish was achieved. After taking photos of the final result, the patient was given oral hygiene maintenance instructions, and he was put under recall appointment after six months.

\section{DISCUSSION}

Comparing the preoperative and postoperative photos, we were able to assess to what extent the aesthetic effect was improved by the prosthetic treatment. We managed to approach the proportional number indicating the mesiodistal width of the teeth compared with each other, viewed from the front, with the described values of an ideal denture (golden proportion, $62 \%$ ), i.e. we have managed to shrink the $86 \%$ visibility of the right lateral incisor compared to the central incisor to $72 \%$, and the $66 \%$ visibility of the left lateral incisor compared to the 


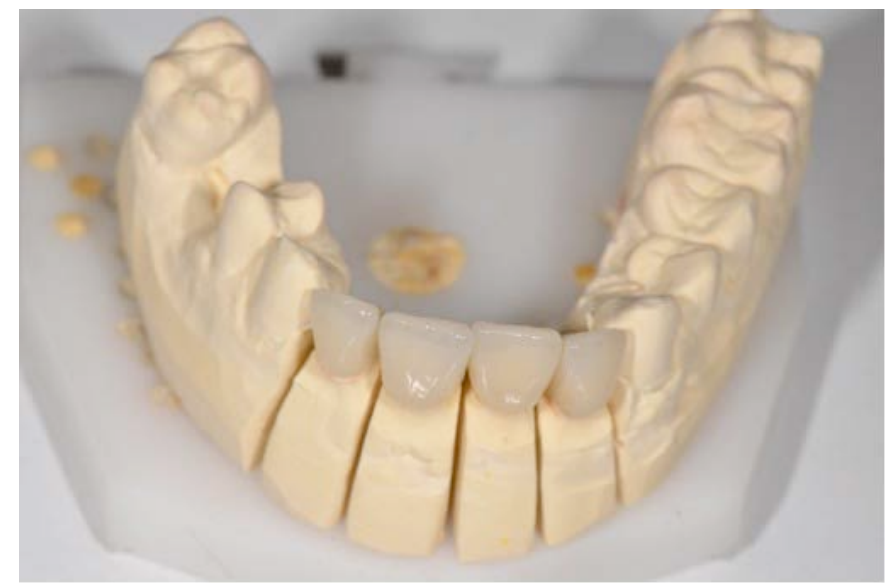

FIGURE 4. IPS e.max crowns

central incisor to $63 \%$. The difference between the measured values does not seem considerable, nevertheless, even with these values, a significant aesthetical difference can be seen in the outcome.

We have managed to bridge over the $6 \mathrm{~mm}$ difference found during the model analysis between the available and the necessary space, decreasing the mesiodistal width of the crowns prepared for the four upper incisors and through the slightly noticeable rotation of the crowns. When decreasing the width of the crowns for the upper central incisors, we took into consideration the proportion between the width and height characteristic to the ideal denture. ${ }^{4,9}$ The proportion between the width and height of the crowns was $75 \%$, which complies with the aesthetical requirements. ${ }^{13,14}$

This decrease in measure was not yet enough, for the crowns to fit in the given narrow place, therefore we solved the remaining lack of space with a slight rotation of the teeth, by this we have managed to establish an effect identical to the natural teeth (Figure 5).

The correction of the irregular curvature of the maxillary incisal edges contributes to the formation of an aesthetic smile, which can be seen as the outcome of the treatment. ${ }^{5,11,12}$

\section{CONCLUSION}

During the prosthetic planning, these measurements may serve as a guideline to the dentist. The judgement of what we consider an aesthetic smile or denture, respectively, is totally subjective. The ideal values we used in certain measurements are considered by several studies as characteristic for aesthetic dentures, and thus, during planning they

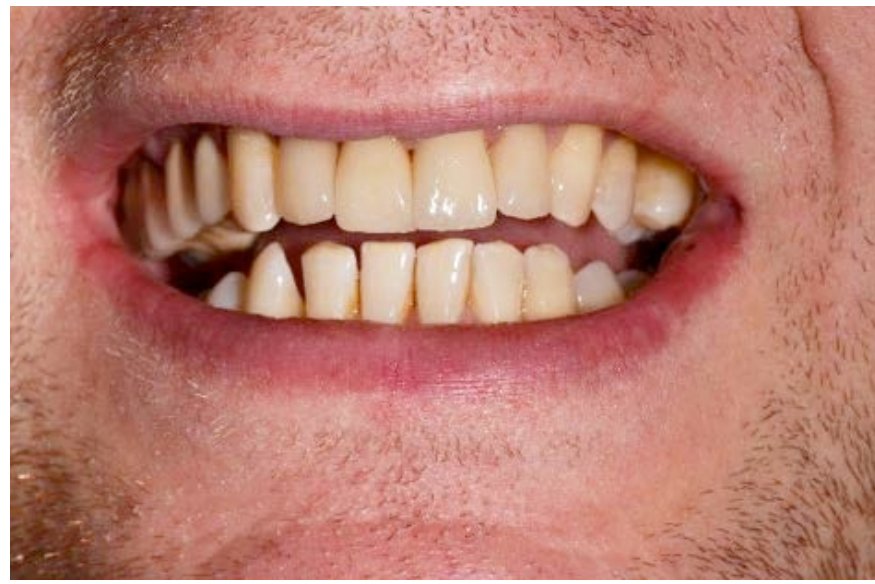

FIGURE 5. Postoperative photograph

may serve as an objective basis for the formation of an aesthetic denture and a more aesthetic smile.

\section{CONFLICT OF INTEREST}

Nothing to declare.

\section{REFERENCES}

1. Parakash V, Gupta R. Instant Orthodontics - A Modality to Correct Malaligned Teeth. Journal of Clinical and Diagnostic Research. 2010;4:29472970.

2. Heymann HO, Kokich VG. Instant Orthodontics: viable treatment option or "quick fix" copout? J Esthet Restor Dent. 2002;14:262-264.

3. Kim J, Chu S, Gurel G, Cisneros G. Restorative Space management: treatment planning and clinical considerations for insufficient space. Prac Proced Aesthet Dent. 2005;17:15-25.

4. Snow SR. Esthetic smile analysis of anterior tooth width: The golden percentage. J Esthet Dent. 1999;11:177-184.

5. William RP, Henry WF, David MS. Contemporary orthodontics. In: William RP, David MS, James LA. Orthodontic Diagnosis: The Development of a Problem List. 4th ed. St. Louis/Missouri: Mosby Elsevier; 2007. p. 185-200.

6. Levin El. Dental esthetics and the golden proportion. J Prosthet Dent. 1978;40:244-252

7. Levin El. The updated application of the golden proportion to dental aesthetics. Aesthetic dentistry today. 2011;5:22-27.

8. Preston JD. The golden proportion revisited. J Esthet Dent. 1993;5:247251.

9. Cooper GE, Tredwin CJ, Cooper NT, Petrie A, Gill DS. The influence of maxillary central incisor height-to-width ratio on perceived smile aesthetics. Br Dent J. 2012;212:589-599.

10. Karamifar A, Nazari M, Hasani A. The golden proportion in dentistry: a literature review. Middle East Journal of Rehabilitation and Health. 2016;3:e34020.

11. Borzabadi-Farahani A. A review of the evidence supporting the aesthetic orthodontic treatment need indices. Prog Orthod. 2012;13:304-313.

12. Camara CA. Aesthetics in Orthodontics: six horizontal smile lines. Journal of Orthodontics. 2010;15:118.

13. Magne P, Gallucci G, Belser UC. Anatomic crown width/length ratios of unworn and worn maxillary teeth in white subjects. J Prosthet Dent. 2003;89:453-461.

14. Magne P, Belser $U$. Bonded porcelain restorations in the anterior dentition: A Biomimetic Approach. Quintessence Pub. Co. Germany, 2002. p. 158159. 